\title{
Gut microbiome and cellular senescence in the context of aging and disease: an emerging frontier
}

\author{
Rohit Sharma*, Ph.D. \\ *Faculty of Applied Sciences \& Biotechnology, Shoolini University, Solan, 173229, India.
}

Correspondence to lead author: Dr. Rohit Sharma

Assistant Professor (INSPIRE Faculty Fellow)

Faculty of Applied Sciences \& Biotechnology, Shoolini University, Solan, 173229, India.

rohit25sharma@gmail.com

rohit.sharma@shooliniuniversity.com

ORCID: 0000-0001-6209-3845.

\begin{abstract}
The significance of diversity, composition, and functional attributes of the gut microbiota is recognized in human health and disease. Studies have also shown that the gut microbiota is related to human aging, and a causal relationship between gut microflora dysbiosis and chronic age-related disorders is also becoming apparent. Further, emerging evidence indicates that age-associated changes in the gut microbiome are predictors of human survival and longevity. Recent advances in our understanding of the cellular and molecular aspects of biological aging have revealed a cellular senescence-centric view of the aging process. However, the association between gut microbiome and cellular senescence is only beginning to be understood. The present review provides an integrative view of the emerging relationship between the gut microbiome and cellular senescence in aging and disease. Evidence relating to microbiome-mediated modulation of senescent cells, as well as senescent cells-mediated changes in intestinal homeostasis have been discussed. Unanswered questions and future research directions have also been deliberated to truly ascertain the relationship of the gut microbiome and cellular senescence for developing microbiome-based age-delaying and longevity promoting therapies.
\end{abstract}

Keywords: Aging; Microbiome; Probiotics; Cellular senescence; SASP; Stress; Immunity 


\section{Introduction}

Human beings are considered 'metaorganisms' due to our close symbiotic relationship with microorganisms which account for at least half the number of total cells in our body (Sender et al., 2016). Most of these microbes are found in the gut, and extensive research has established their central role in maintaining human health and systems by regulating the development and modulation of immune responses (Schluter et al., 2020), host nutrition and metabolism (Goodrich et al., 2016; Kaoutari et al., 2013), brain development and behavior (Smith, 2015) as well as progression of diseases such as cancer (Sobhani et al., 2019) and diabetes (Li et al., 2020a). Moreover, the identification of an association between altered gut microbiota composition and the magnitude of COVID-19 severity in the ongoing pandemic highlights the importance of gut microflora in shaping bodily response to novel infectious agents (Yeoh et al., 2021). Gut microbiota is abundant in complexity and diversity, and a number of different microorganisms such as archaea, bacteria, fungi, and viruses reside in human gastrointestinal tract (Ferreiro et al., 2018). The microbiota is maternally acquired at birth (Dominguez-Bello et al., 2010) and undergoes changes throughout the lifespan dominated by dietary habits and environmental signals (O'Toole and Claesson, 2010). Gut microbial heterogeneity amongst communities has also been observed with implications in health and disease (Das et al., 2018; Priya and Blekhman, 2019).

Aging has been described as 'an unresolved problem of biology'(Medawar, 1952), although intensive studies in the past few decades have vastly improved our understanding of the causes and effects of aging. In general, nine 'hallmarks of aging' have been identified (López-Otín et al., 2013) which ultimately augment the principle that aging is essentially a time-dependent culmination of various cellular and biochemical damage (Gems and Partridge, 2013; Report, 2021). Cellular senescence, a physiological response to stress, is emerging as one of the most well-studied hallmark of aging (Borghesan et al., 2020), and gradual accumulation of senescent cells (SC) in tissues and organs is likely accountable for increased risk of disease and mortality (Baker et al., 2016). Apart from rodents and humans, age-dependent exponential increase in the numbers of SC accompanied by activation of characteristic DNA damage response has also been observed in primates (Herbig et al., 2006). Further, a breakthrough study demonstrated that removal of SC burden in various aging tissues has the potential to delay the development and severity of age-related pathologies and improve lifespan (Baker et al., 2011). Subsequently, molecular targeting of SC using pharmacological interventions showed that SC can be selectively removed from the organism which resulted in lifespan extension and delayed development of age-related symptoms and diseases (Zhu et al., 2015). Since then, several natural and synthetic compounds, which selectively target SC (senolytics) have been identified (Kirkland and Tchkonia, 2020; Salekeen et al., 2021), and senolytics-based clinical trials are already showing promising results in countering some of the deleterious effects of aging (Dolgin, 2020; Hickson et al., 2019; Justice et al., 2019).

An intricate association between the gut microbiome and organismal aging is increasingly becoming apparent (Kim and Benayoun, 2020). Since the gut microbiome coevolves with the host, organismal aging is inevitably accompanied by changes in the gut microbiome (Hopkins and Macfarlane, 2002; Mariat et al., 2009). As a result, distinct differences in microbial composition between young and elderly human subjects have been observed (Claesson et al., 2011; Kong et al., 2016). However, the interrelationship between gut microbiome and cellular senescence in regulating the process of aging and related diseases is only beginning to be understood. The present manuscript provides an overview of cellular senescence and gut microbiota in driving the aging process, and then reviews the available evidence linking the two phenomena. Possible therapies and future directions to 
better understand the causal relationship between cellular senescence and gut microbiota in governing organismal aging are also presented.

\section{Cellular senescence links biological aging}

Cellular senescence is a physiological stress response mechanism in mammalian cells characterized by irreversible cell cycle arrest mediated by activation of cell cycle inhibitor pathways (p53/p21 and/or p16 $\left.{ }^{\text {Ink4a }} / \mathrm{pRb}\right)$, DNA damage, chromatin remodelling, senescenceassociated $\beta$-galactosidase (SA- $\beta$-gal) activity changes in metabolic signalling pathways (mTOR/AMPK), impaired mitochondrial ROS production, cellular hypertrophy and the development of characteristic senescence-associated secretory phenotype (SASP) (Herranz and Gil, 2018; van Deursen, 2014). The connection between aging and cellular senescence was initially based on the observation that SC tend to accumulate in aging tissues (Krishnamurthy et al., 2004). However, SC develop naturally and their crucial role in regulating processes such as wound healing and embryonic tissue remodelling have also been documented (Demaria et al., 2014; Muñoz-Espín et al., 2013). Today, it is accepted that agerelated accumulation of SC, and not SC per se, is responsible for the increased age-related pathologies and decreased healthspan and lifespan (He and Sharpless, 2017). It is argued that cellular senescence evolved as a mechanism to protect against tumorigenesis in cells exposed to chronic stress-induced damage (Sager, 1991), as opposed to apoptosis which is activated in response to acute stressors (Childs et al., 2014; Spallarossa et al., 2009). In younger organisms, the SASP factors secreted by SC chemotactically attract the cells of the immune system such as macrophages, NK cells or cytotoxic T cells which then recognize SC through specific cell surface receptors ultimately resulting in their elimination (Song et al., 2020). As organisms age, a tissue-specific non-linear accumulation of SC is observed that eventually impairs organ functions (Idda et al., 2020; Tuttle et al., 2020). The chronic secretion of SASP components, which are a milieu of several cytokines and growth factors, by accumulating SC plays a particularly deleterious role by rendering harmful bystander effects on nearby healthier cells ultimately promoting pro-inflammatory and pro-tumorigenic behaviour (Nelson et al., 2012) (Figure 1).

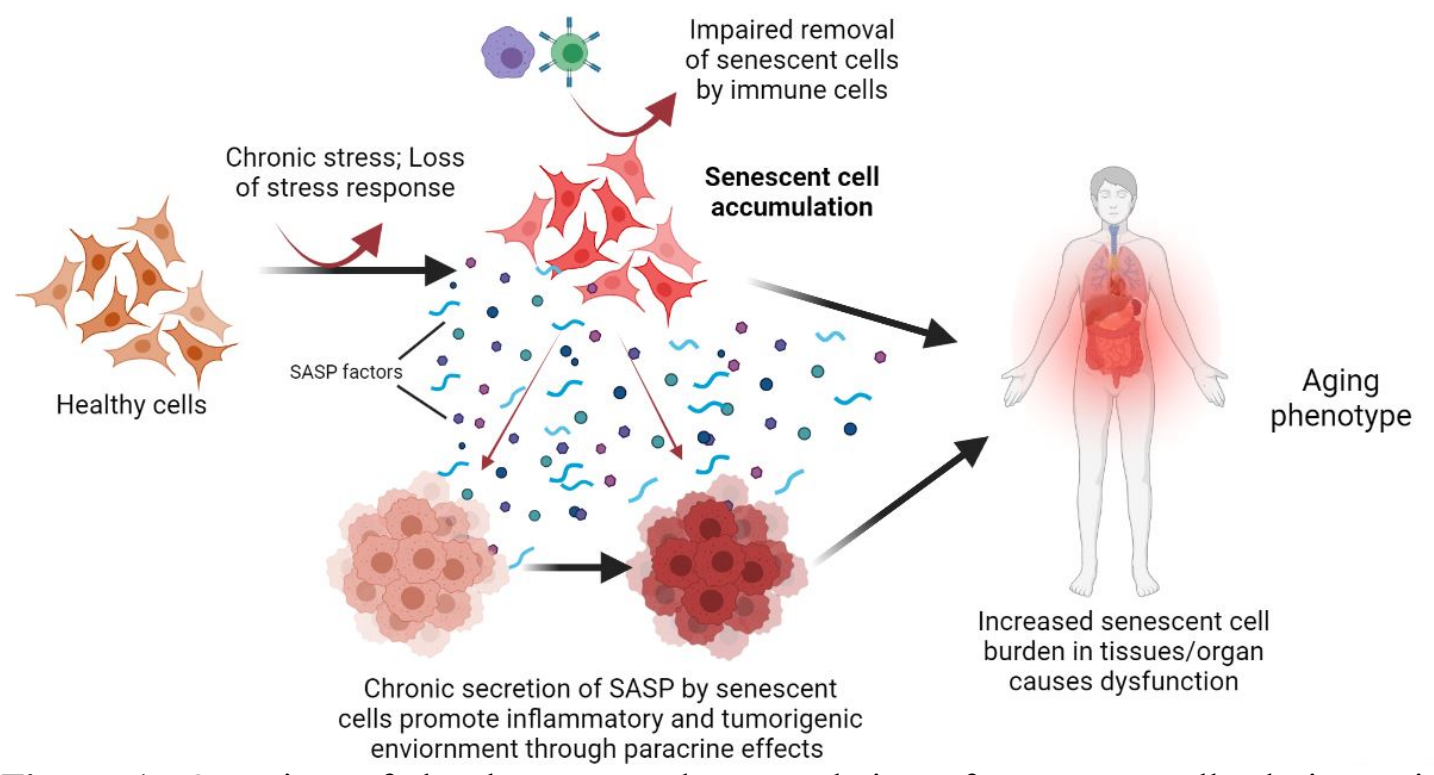

Figure 1: Overview of development and accumulation of senescent cells during aging. Chronic stressors (oxidative/replicative/oncogenic), reduced cellular stress response capacity, and impaired immune functions can augment the development and accumulation of senescent 
cells in various tissues. Persistent senescent cells develop SASP which affect nearby healthier cells through paracrine mechanisms, promote inflammatory and tumorigenic environment and ultimately impair organ functions, and promote the aging phenotype. Illustration created at app.biorender.com.

Thus, SC, which are evolutionarily considered anti-tumorigenic, eventually render the aging organism more prone to cancerous and inflammatory disorders in a classic case of antagonistic pleiotropy (Campisi, 2005). Corroborating this, a wealth of data have shown that targeting the development of cellular senescence during aging improves longevity and healthspan, suppresses inflammatory disorders, enhances organ functioning, while transplantation of SC can provoke disease condition in healthy animals (Baker et al., 2011; Cai et al., 2020; Kim et al., 2020; Xu et al., 2017; Xu et al., 2018).

The development of cellular senescence is intimately linked to cell intrinsic and extrinsic stressors (de Magalhães and Passos, 2018). Cell autonomous (intrinsic) stress could be attributed to replicative stress, mitochondrial dysfunction, autophagic dysregulation and proteostasis, while external stressors include metabolic stress, heat, genotoxic agents, heavy metals etc. It has been shown that during aging, the natural response to stress is impaired (Chen et al., 2020a), which, combined with the chronic exposure to stressors result in perturbations in mitochondrial redox balance, energy production and aggravation of inflammatory pathways (Bulua et al., 2011). This can result in a vicious loop wherein damaged mitochondria produces more free radicals, and this sustained redox pressure further damages macromolecules and initiate DNA damage response that propels cells into the state of permanent cell cycle arrest and SASP thereby hampering tissue and organ functions characteristic of aging (Childs et al., 2014) (Figure 1). It is thus no surprise that premature stress-induced models of cellular senescence have been successfully used in in vitro studies. While other stressors may be indefinite and subject to regulation, replication-induced stress is a fundamental feature of mitotic cells that gradually contributes to the development of replicative cellular senescence (Campisi, 1997). Replicative stress arises because during each cell division, chromosomes lose a part of their protective telomere cap, and with each subsequent division, telomeres become too short to sustain further replication resulting in activation of cellular senescence program (Campisi, 1997). However, there is evidence that oxidative stress can also accelerate telomere attrition suggesting that chronic presence of different stressors can rapidly induce/augment different (oxidative/replicative/oncogenic) senescence program in cells (Epel et al., 2004; Tchirkov and Lansdorp, 2003).

The exact causes for age-dependent increase in tissue SC burden are not completely understood. Recent studies have shown that accumulation of SC in tissues is not linear, but accelerates with age, suggesting that a threshold barrier may exist beyond which SC accumulation exponentially rises (Liu et al., 2009; Tuttle et al., 2020). The immune system is rapidly emerging as critical regulator of apparent age-associated $\mathrm{SC}$ accumulation. A recent study conclusively demonstrated that immune cells with dysfunctional cytotoxic ability exhibit inefficient clearance of SC in vivo resulting in their accumulation and augmentation of inflammatory disorders (Ovadya et al., 2018). This study advanced the idea that deleterious widespread functional and phenotypic changes in aging immune cells (immunosenescence) can result in impaired immunosurveillance and cytotoxic effector functions that may promote SC accumulation (Song et al., 2020). However, the situation gets further compounded by the fact that similar to other cells, immune cells also undergo cellular senescence with age, and thus their efficacy in clearing SC may be subject to regulation by both immunosenescence and cellular senescence (Burton and Stolzing, 2018; Yousefzadeh et al., 2021). Surprisingly, a recent study demonstrated that similar to tumorous cells, SC can also develop immune evasive functions which may augment their survival and persistence in 
tissues thereby indicating a deeper relationship between host immunity and SC (Pereira et al., 2019). Taken together, it is evident that accumulation of SC is harmful, and strategies aimed at prevention or regression of cellular senescence during aging are desirable to alleviate morbidity and mortality in elderly.

\section{Gut microbiota in aging and associated diseases}

The diversity and composition of the gut microbiota is altered with age, which, in turn affects metabolism and immune regulation that predispose elderly to inflammatory disorders (Bana and Cabreiro, 2019; Zhang et al., 2020). Therefore, maintenance of homeostatic balance in the intestinal flora is beneficial for the host, and any perturbations in microbial community structure that induce an imbalance between commensal gut microbes and opportunistic pathogens can render the gut vulnerable to damage and loss of intestinal homeostasis. The altered gut microbial state or 'dysbiosis' can be attributed to loss of beneficial organisms, proliferation of harmful pathogenic genera or overall decrease in microbial diversity (DeGruttola et al., 2016). For instance, studies have shown that agerelated gut dysbiosis generally results in enhanced growth of facultative anaerobes, decreased numbers of beneficial probiotic bacterial species and an altered firmicutes/bacteroidetes ratio (Salazar et al., 2017; Vaiserman et al., 2020). There is enough evidence available to suggest a correlation between gut dysbiosis, longevity and the prevalence of chronic disorders (Cardenas et al., 2014; Kamo et al., 2017; Lee et al., 2019). In fact, a recent report highlights that gut microbiome composition is a predictor of human survival and increased abundance of Bacteroides or low gut microbiome uniqueness is associated with decreased lifespan and enhanced morbidity (Wilmanski et al., 2021). Based on machine learning, another recent report inferred that age-related changes in the gut microflora can be used to precisely predict the age of an individual (Galkin et al., 2020). Further, dysbiosis in the commensal bacterial population resulted in shortening of lifespan in Drosophila (Iatsenko et al., 2018), while colonizing the gut of middle-age African turquoise killifish with bacteria isolated from young donors resulted in lifespan extension and delayed behavioral decline (Smith et al., 2017). Microbiota transplantation studies have demonstrated that altered gut microbiome of elderly is sufficient to induce morbidity in young recipients. For instance, it was observed that transplantation of the gut microbiota from aged mice to germ free mice promoted inflammation in the small intestine and enhanced the leakage of inflammatory bacterial components into circulation which were correlated with higher levels of TM7 bacteria and Proteobacteria (Fransen et al., 2017). A recent work showed that fecal microbiota transplantation from aged murine donors led to impaired spatial learning and memory in young adult recipients accompanied by a strong reduction of short-chain fatty acids (SCFAs) producing bacteria such Lachnospiraceae, Faecalibaculum, and Ruminococcaceae (D'Amato et al., 2020). Similarly, fecal transplant gavages from old mice (but not young mice) to germ free mice showed decreased fecal SCFAs production, and recipient animals demonstrated depressive-like behavior, impaired short-term memory, and spatial memory suggesting that aged microbiome alone is sufficient to decrease SCFAs in the host and to produce cognitive decline (Lee et al., 2020). Similarly, fecal microbiota transplantation from old to young rats impaired cognitive behavior, decreased regional homogeneity, changed synaptic structures, increased glycation-end products and enhanced oxidative and inflammatory stress in recipient young animals (Li et al., 2020b). A single-center clinical trial recommended fecal microbiota transplantation as a home therapy which could clinically benefit recovery in elderly patients with Clostridium infection (Jørgensen et al., 2020). On the contrary, it has been reported that transplanting the gut microbiota of healthy old donor mice into young germ-free recipient mice enhanced hippocampal neurogenesis, intestinal growth, and activated the longevity FGF21-AMPK-SIRT1 signalling pathways in the liver which was attributed to the increased 
presence of butyrate producing microbes (Kundu et al., 2019). In this regard, another study shown that although aging in healthy donors is associated with compositional alterations in the faecal microbiome, but the overall microbial diversity and effectiveness of faecal microbiota transplantation may still be preserved (Anand et al., 2017). However, despite heterogenic variations in different experimental conditions, it is evident that beneficial effects of desirable bacterial populations, such as butyrate-producing microbes, may be useful in protection against age-associated morbidity. Aging not only influences the gut microflora composition, but is also associated with changes in the gut lumen and barrier functions such as shrinkage of the protective mucus layer of the gut, loss of intestinal tight junction proteins, and increased permeability of the epithelial barrier (Elderman et al., 2017; Tran and Greenwood-Van Meerveld, 2013). As a result, bacteria and their metabolites can escape the gut lumen which may provoke immune cells in the Peyer's patches of lamina propria, and further augment systemic inflammation if translocated into peripheral circulation (Thevaranjan et al., 2017). Overall, age-dependent changes in the gut microbiome composition, remodeling of the intestinal epithelial barrier, and loss of intestinal permeability disrupt intestinal homeostasis and can result in a systemic pro-inflammatory environment with implications in the pathogenesis of several inflammatory disorders (Andersen et al., 2017) (Figure 2).

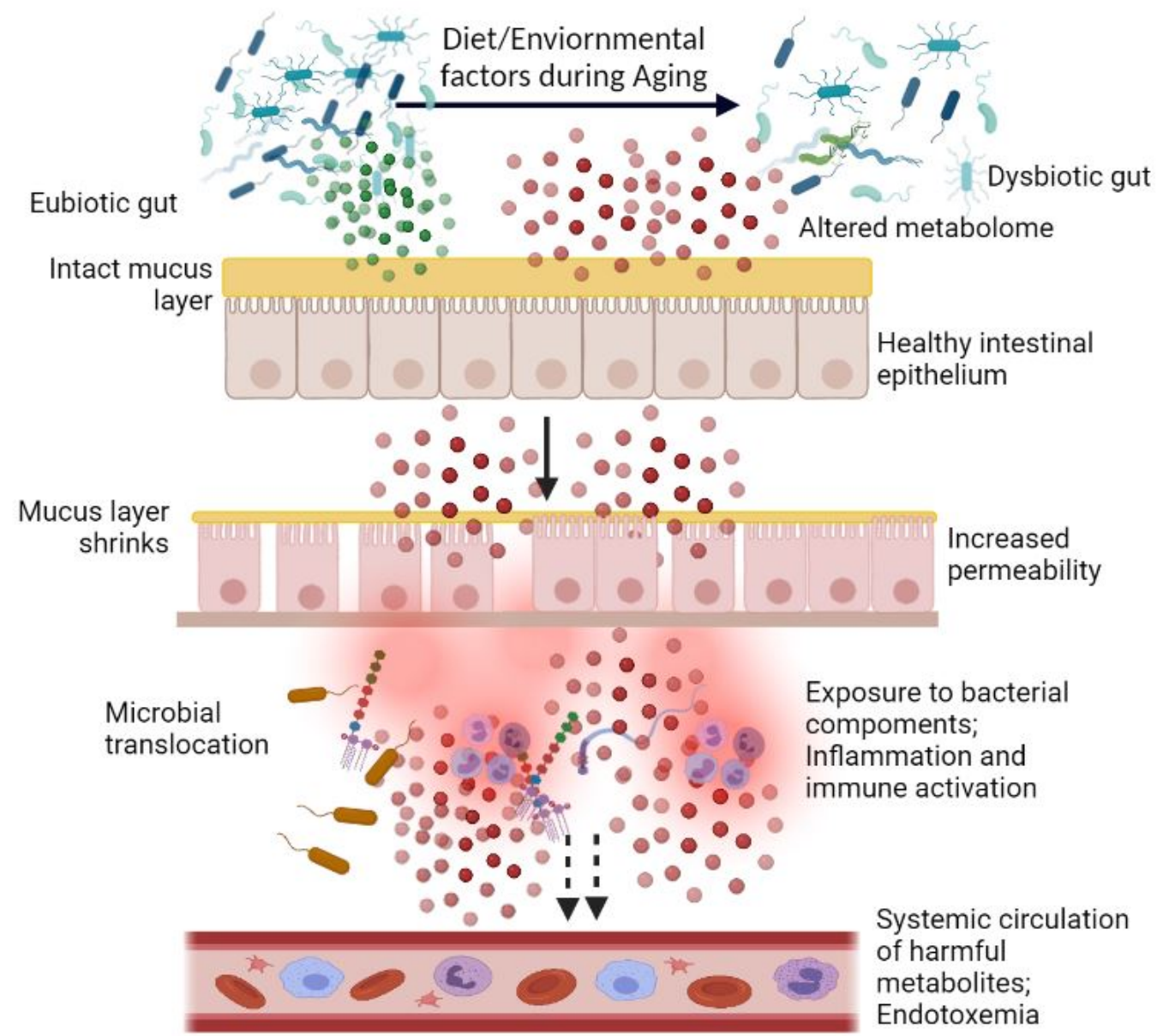

Figure 2: Schematic diagram depicting the influence of aging on gut homeostasis. Dominated by diet and environmental factors, aging induces structural and functional changes in the gut microflora resulting in a dysbiotic gut. Altered metabolome of the dysbiotic gut, shrinkage of protective mucus layer and loss of tight junction proteins promote 
'leakage' through the gut barrier. Bacteria as well as bacterial components, such as LPS and flagellin, can translocate through the 'leaky gut' and reach lamina propria resulting in activation of immune cells in the Peyer's patches, and can also cause systemic inflammatory aggravation through peripheral circulation. Illustration created at app.biorender.com.

The role of altered gut microbiota composition and the development of age-related chronic diseases such as immunosenescence, inflamm-aging, cancer, diabetes, and cardiovascular disorders is also recognized (Buford, 2017). A recent study demonstrated that gut microbiota changes in young adulthood (32-42 years age) may be associated with major metabolic, immunological and inflammatory parameters of aging, which could be useful in detecting accelerated aging (Renson et al., 2020). Microbiota and the host immune system have co-evolved to allow the existence of commensal bacteria through immune tolerance, while a robust immune response is generated against foreign pathogenic agents (EloeFadrosh and Rasko, 2013). Moreover, gut microbiota is critical for appropriate development and maturation of host immunological responses (Chung et al., 2012), and there is also evidence that the deleterious effects of immunosenescence may also be linked to abnormal immune response against gut microbiota due to dysbiosis or decreased tolerance (Guigoz et al., 2002). A study on healthy middle-aged and old-aged human subjects showed a quantitative and qualitative correlation between the composition of the gut microflora and the markers of immunosenescence (Shen et al., 2018). A recent report demonstrated that exposing aged mice to the microbiota from young mice dramatically improved M-cell maturation in the intestine of the aged mice and increased antigen uptake and IgA responses suggesting novel role of gut microbiota in enhancing M-cell mediated immunosurveillance and vaccine efficacy in elderly (Donaldson et al., 2020). We have previously observed that gradual aging manifests several aspects of immunosenescence in aging mice which can be alleviated by the consumption of probiotic fermented milk that resulted in reduced inflammaging, suppression of Th1/Th2 immune imbalance and improved response to pathogenic Escherichia coli (Sharma et al., 2014). The risk of type II diabetes increases with age, and changes in the gut microbiome can modulate the physiological effects of diabetes by affecting glucose metabolism, energy expenditure, inflammation and gut permeability (Gurung et al., 2020). Recent studies have revealed that type II diabetes is also associated with altered microbiota composition generally characterized by a decrease in antiinflammatory, probiotic and potentially pathogenic species of bacteria (Almugadam et al., 2020; Das et al., 2021). However, due to the heterogenic, multifactorial and multi-organ nature of type II diabetes, conclusive causal evidence implicating the involvement of specific microbes in the pathogenesis of the disease is challenging and remains elusive. Host microbiome is also rapidly emerging as a predictor of severity and therapy outcomes in several incidences of cancers (Poore et al., 2020; Zhuang et al., 2019). Numerous studies have shown altered gut microbioata composition during the development of various cancers including but not limited to colorectal cancer (Nakatsu et al., 2015), gastric cancer (Ferreira et al., 2018), lung cancer (Zhuang et al., 2019), pancreatic cancer (Half et al., 2019), prostate cancer (Daisley et al., 2020), breast cancer (Goedert et al., 2015), brain cancer (D'Alessandro et al., 2020) and even leukemia (Vicente-Dueñas et al., 2020). Mechanistically, the gut microbiome can influence tumorigenesis at sites distant to the gut by regulating circulatory levels of metabolites and nutrients as well as through the release of microbial toxins which are currently under intense investigation (Parida and Sharma, 2021). Changes in the gut microbiota community has also been documented in the pathogenesis of age-related cardiovascular disorders such as heart failure and coronary artery diseases. In general, decreased abundance of butyrate producing bacteria such as Faecalibacterium, Roseburia and Eubacterium rectale has been observed in patients with coronary heart disease (Jie et al., 2017; Karlsson et al., 2012; Zhu et al., 2018), as well as in patients with chronic heart failure 
and cardiac insufficiency (Cui et al., 2018; Kamo et al., 2017; Luedde et al., 2017). While the mechanisms of the pathogenesis of gut microbiome dysbiosis in generation of cardiovascular disorders is yet unclear; age-related increase in intestinal permeability and subsequent increased diffusion of harmful bacterial metabolites that result in sustained inflammation have been considered. For example, increased circulatory levels of microbe-dependent trimethylamine-N-oxide has been observed in patients with heart failure which was also correlated with increased risk of mortality and the development of cardiovascular disorders (Tang et al., 2014; Wang et al., 2011).

\section{Gut microbiome and cellular senescence}

A bidirectional relationship between the gut microbiome and cellular senescence can be envisaged wherein secretory metabolites of the gut microbiota and/or gut microflora mediated bio-transformed dietary components can directly impact cellular senescence in intestinal cells, while the accumulating senescent epithelial cells in the intestine (and SASP thereof) may contribute to altered cellular functions and immune activation (Figure 3).
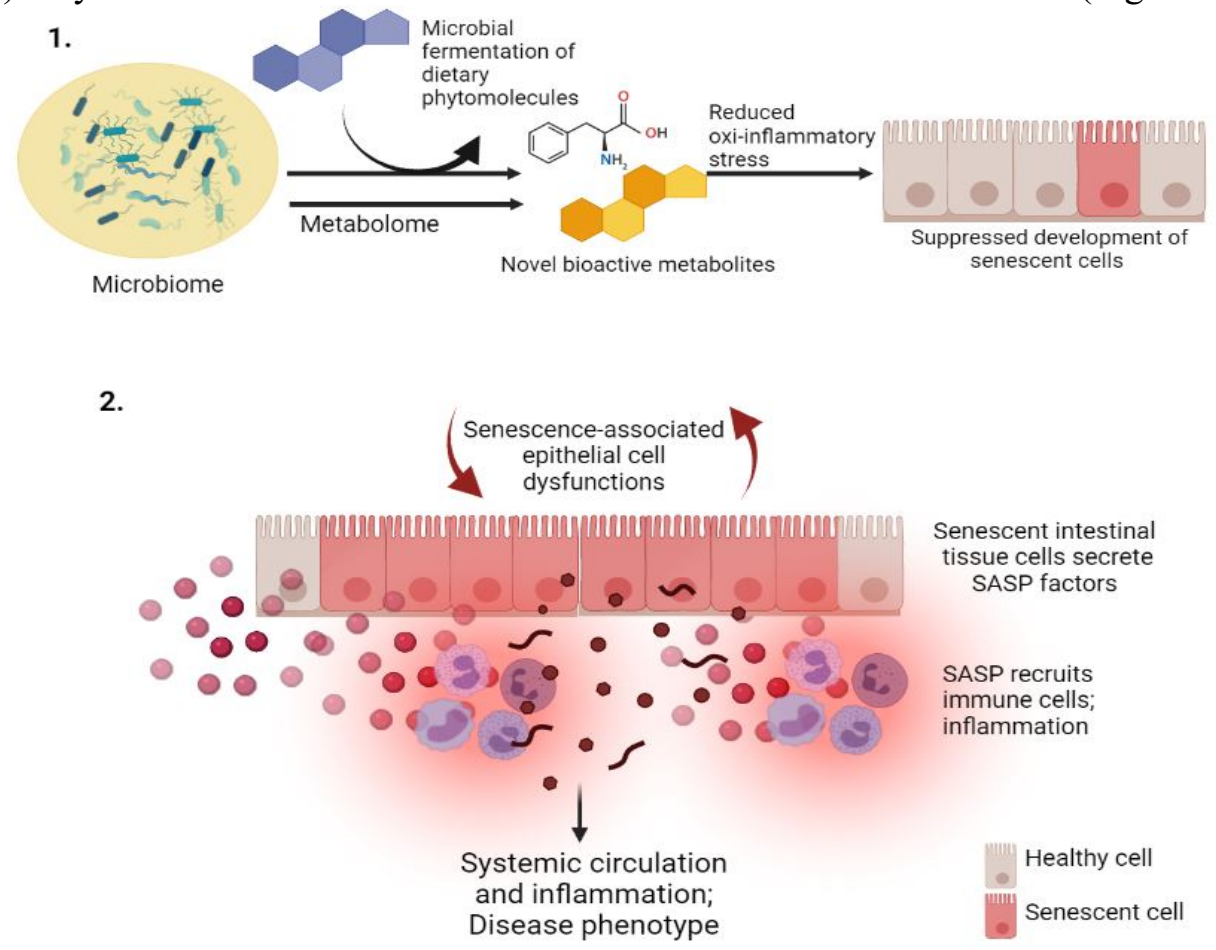

Figure 3. Relationship between the gut microbiome and senescent cells. (1) Metabolites of the microbiome or those produced by microbial biotransformation of dietary components, can directly influence cellular stressors and stress response capacity which can ultimately attenuate the development and progression of cellular senescence. (2) Cellular senescence can contribute to functional dysfunctions in the intestinal cells, and SASP secreted by these cells can augment aberrant intestinal inflammation and immune cell activation. Illustration created at app.biorender.com.

The gut microbiome utilizes dietary fuel to synthesize an array of bioactive metabolites such as SCFAs, phenols, neurotransmitters, HPA hormones, endotoxins, ammonia etc. through processes such as microbial fermentation (Oliphant and Allen-Vercoe, 2019). These metabolites can reach circulation and affect the functioning of distal organs and systems (Agus et al., 2021; Colldén et al., 2019), and as such, the gut microbiome is often considered as a complex virtual endocrine organ (Clarke et al., 2014). Although cellular 
senescence is subject to several regulations, however, the natural development of cellular senescence can be regulated by cells' ability to counter ROS mediated oxidative damage and stress response capacity (Colavitti and Finkel, 2005). Indeed, it has been demonstrated that increased oxidative stress over the lifespan of an animal can directly enhance the accumulation of SC (Zhang et al., 2017), and application of antioxidants can attenuate cellular senescence both in vitro and in vivo (Kumar et al., 2019a; Varela-Eirín et al., 2020). Therefore, it is conceivable that the development and accumulation of SC can be attenuated or delayed either by enhancing the potency of cellular stress response and/or redox balance, or simply by neutralizing the external oxidative/inflammatory stressors. In this regard, diverse metabolites of the gut microbiome and probiotic bacteria, or microbiome-fermented dietary phytomolecules are known to exert strong anti-inflammatory and antioxidant attributes which may be useful in preventing senescence-associated pro-inflammatory and pro-tumorigenic environment (Chang et al., 2014; Chung et al., 2020; De Marco et al., 2018; Riaz Rajoka et al., 2019; Rossi et al., 2020; Wang et al., 2018) (Figure 3). Moreover, complex phytomolecules, especially polyphenols, which are poorly absorbed in the small intestine, are fermented by the colon microbiota to yield several simpler molecules which may have different and/or enhanced biological effects than the original compound (Parkar et al., 2013; Sharma and Padwad, 2020c; Wang et al., 2018). We have also observed that amalgamation of a probiotic bacteria and tea polyphenol EGCG can differentially modulate the aging immune system as compared to individual treatments of constitutive elements (Sharma et al., 2019). However, our recent work provided first evidence of stress-induced premature cellular senescence suppression by the application of probiotic bacteria. It was observed that metabolites secreted by a probiotic Lactobacillus fermentum, isolated from human fecal matter, can attenuate multiple characteristic markers of senescence in preadipocytes such as cellular hypertrophy, SA- $\beta$-gal activity, induction of SASP, DNA damage response, Akt/mTOR pathway and activation of cell cycle inhibitor signaling (Kumar et al., 2020). Using a reconstructed skin model, another study reported that extract of bacterium Sphingomonas hydrophobicum can delay skin senescence by attenuating the activation of $\mathrm{p} 21, \mathrm{p} 16^{\text {Ink4a }}$ cell cycle inhibitors and SA- $\beta$-gal activity resulting in restructuring of skin as well as psychobiological effects (Gervason et al., 2019). It is thus conceivable that secretory factors of the gut microbiota can influence cellular senescence, although further in vivo studies are warranted.

Intestinal epithelial cells (IECs) not only act as protective barrier that separate the host tissues from the luminal environment, but also serve as sensors to maintain inflammatory homeostasis in the gut. Age-related progressive increase in SC burden and chronic SASP environment in the intestinal tissue can dysregulate the normal functioning of IECs (such as barrier functions) and ultimately contribute to increased gut permeability and enhanced susceptibility to inflammation and infections (Figure 3). A recent analysis showed agedependent increase in senescent $\mathrm{p} 16^{\text {Inka4a}}+/ \mathrm{p} 21+$ cells in various human organs, including the colon tissues, suggesting SC accumulation as a function of intestinal aging across the human lifespan (Idda et al., 2020). Similarly, another report revealed that intestinal tissue developed strong signatures of cellular senescence as evident by increased expression of senescence markers-p16 ${ }^{\text {Ink4a }}, \mathrm{p} 21^{\text {Cip } 1}$ and SA- $\beta$-gal activity both in WT mice and accelerated aging mice model- Ercl $^{-/ \Delta}$ (Yousefzadeh et al., 2020). We also observed an age-dependent increase in DNA damage, cellular senescence $\left(\mathrm{p} 53 / \mathrm{p} 21^{\mathrm{WAF} 1}\right)$, activation of SASP regulators (NFkB, p38MAPK, Cox-2) and metabolic stress in the intestinal tissue of aged mice indicating their vulnerability to spontaneous age-related genotoxic stress (Sharma et al., 2021). In addition, intestinal epithelial organoids derived from aged mice also showed consistent upregulation of senescence markers such as SA- $\beta$-gal activity and p21 as compared to organoids derived from younger organisms (Uchida et al., 2018). Intestinal epithelial stem cells of old mice 
expressed enhanced mRNA levels of genes associated with cellular senescence and oxidative stress (Moorefield et al., 2017), and radiation exposure also induced premature cellular senescence and SASP phenotype in intestinal stem cells in vivo (Kumar et al., 2019b). It is thus evident that intestinal epithelial as well as stem cells exhibit age-dependent cellular senescence which can contribute to known functional alterations and disruption of gastrointestinal homeostasis. Moreover, the chronic SASP secreted by senescent intestinal cells can promote inflammatory environment and/or oncogenic transformation which can have harmful effects on gut permeability, immune activation as well as the gut microbiome composition (Kumar et al., 2019b; Sharma et al., 2021) (Figure 3). A breakthrough study demonstrated the effects of a dysbiotic gut in the development of SASP and its deleterious effects. It was observed that obesity-induced alterations in the gut microbiota can augment the synthesis of a genotoxic bacterial metabolite- deoxycholic acid, which provokes SASP phenotype in stellate hepatic cells, ultimately inducing pro-inflammatory environment and aiding in the development of hepatocellular carcinoma (HCC) in mice (Yoshimoto et al., 2013). Remarkably, blocking DCA production or antibiotic mediated reduction in gut bacteria efficiently prevented HCC development in obese mice (Yoshimoto et al., 2013). It is also worth noting that although cellular senescence is seemingly undesirable in the context of aging; yet pro-senescence strategies are often considered favorable for preventing the proliferation of cancerous cells (Chen et al., 2005). In this context, bacterial metabolites such as butyrate have shown the potential to limit the proliferation of cancerous cells by upregulating the expression of pro-senescence genes such as p21, p27 and SASP regulators (Thangaraju et al., 2009; Wei et al., 2016), and suppressing genotoxic risk factors of cancer development (Abrahamse et al., 1999; Ebert et al., 2003).

A wealth of emerging data has demonstrated that nutraceuticals, especially phytomolecules can attenuate several aspects of cellular senescence and SASP (Sharma and Padwad, 2020a). Given that these dietary components first interact with the gut microbiome, it is plausible to envisage that the known anti-cellular senescence or other age-associated health beneficial effects of nutraceuticals may also be related to the modulation of gut microbiome and gut metabolites thereof (Sharma and Padwad, 2020b; Sharma and Padwad, 2020d). For instance, the mechanisms of anti-cellular senescence and senolytic attributes of putative senolytics such as quercetin, EGCG, resveratrol and fistein are incompletely understood, and could be mediated by influencing the composition of the gut microbiome (Saccon et al., 2021). This is especially plausible given the fact that bioactive phytomolecules can promote the proliferation of probiotic bacteria, suppress the growth of pathogenic bacteria, and also act as second generation synbiotics (Sharma et al., 2019; Zhang et al., 2013). A recent report has demonstrated that the consumption of a senolytic combination of Dasatinib and Quercetin resulted in lower SC burden (p16 $6^{\text {Inka4a }}$ and p21 expression) and SASP phenotype (Cxcl1, IL1 $\beta$, IL6, MCP1, and TNF- $\alpha$ expression) in small and large intestine as compared with control mice. Remarkably, specific microbial composition in ileal, cecal, colonic, and fecal regions were also distinctly modulated by $\mathrm{D}+\mathrm{Q}$, suggesting that senolytics might improve health via reducing intestinal senescence, inflammation, and gut microbial dysbiosis in older subjects (Saccon et al., 2021). Our recent report also observed that dietary consumption of another putative senolytic, green tea EGCG, decreased SC burden in multiple tissues which correlated with increased suppression of pathogenic bacteria in the gut microbiome of aged mice (Sharma et al., 2021). Another natural senolytic fistein, which is abundant in numerous fruits and vegetables, can suppress premature ovarian failure (Lin et al., 2020) and age-related Parkinson's disease by regulating the composition of gut microbiota (Chen et al., 2020b). Further, polyphenols quercetin and resveratrol can enhance in vivo microbial diversity, especially related to beneficial probiotics such as Lactobacillus and Bifidobacterium, and augment intestinal inflammatory homeostasis (Dos Santos et al., 
2019; Lin et al., 2019; Shi et al., 2020). Similar to polyphenols, few studies have shown that dietary supplementation of probiotic bacteria can also influence inflammatory disorders through the modulation of cellular senescence in colon tissue. For example, administration of Lactobacillus pentosus var. plantarum $\mathrm{C} 29$ to aged rats inhibited inflamm-aging, improved expression of gut tight junction proteins and bacterial diversity which correlated with suppression of cellular senescence in the colon of aged rats (Jeong et al., 2015a). Similarly, consumption of probiotic Lactobacillus brevis OW38 in experimental animals inhibited cellular senescence in colon, and reduced the ratio of Firmicutes or Proteobacteria to Bacteroidetes (Jeong et al., 2016), while consumption of a probiotic mixture IRT5, containing five different probiotic strains, also attenuated multiple aspects of age-dependent colitis in rats by targeting cellular senescence and inflammatory aggravation (Jeong et al., 2015b).

\section{Future research directions}

There are several deficiencies and unanswered questions in our current understanding of the interrelationship between gut microbiota and cellular senescence (Figure 4). Emerging evidence suggests that the gut metabolome could be a more pragmatic and functional marker of a healthy gut (Zierer et al., 2018) since traditional metagenomics-based approaches lack quantitative functional annotation (Frias-Lopez et al., 2008). Recent observations suggest that gut metabolome analyses can effectively reveal microbiome-dependent interactions between diet and metabolites, as well as ethnic and disease conditions (Jain et al., 2019; Tang et al., 2019; Vascellari et al., 2020).

1.

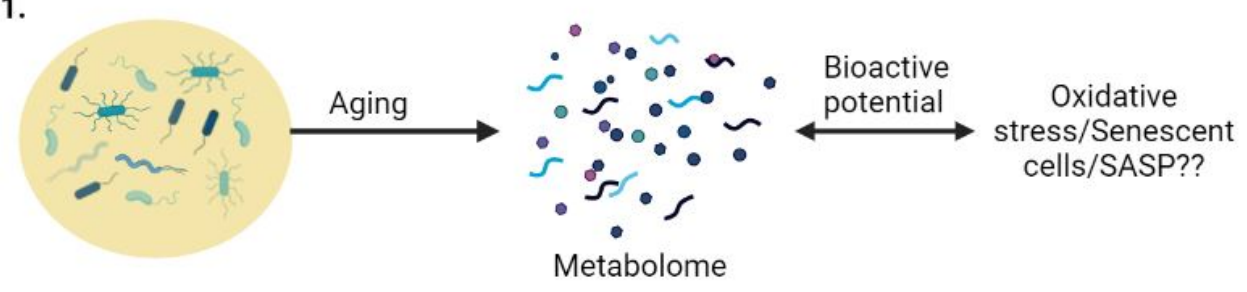

2. Senolytics/Anti-senescence agents/Probiotics

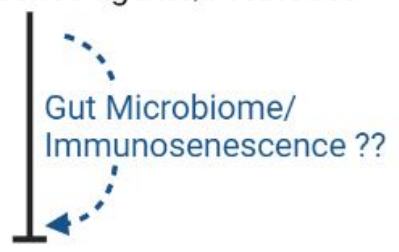

Senescent cell burden

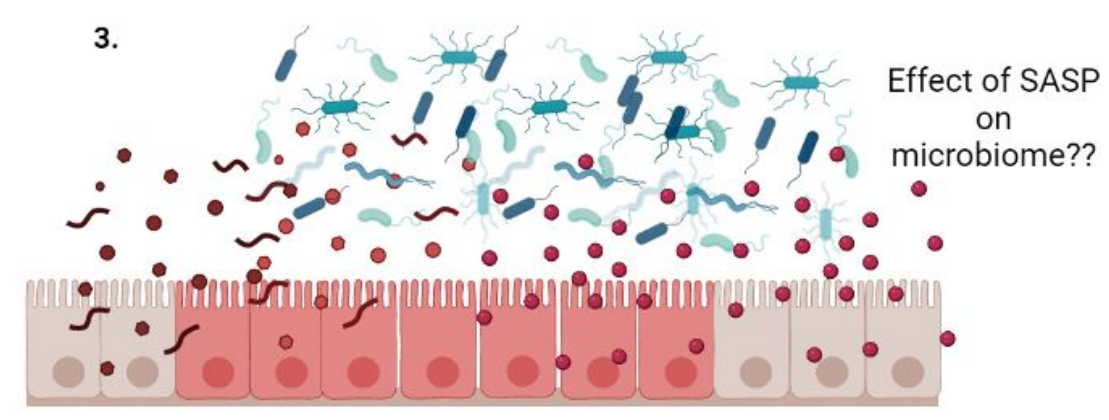

Figure 4. Open questions and opportunities relating gut microbiome and cellular senescence. (1) How the gut metabolome changes with age, and how it could be related to cellular 
senescence and SASP is not known. (2) Whether the known effects of senolytics and antisenescence agents are mediated through gut microbiome modulation is not clear. (3) How does SASP of accumulating senescent cells in the intestine affect the gut microbiome remains unknown. Illustration created at app.biorender.com.

However, studies delineating the role of gut metabolome alterations with lifespan and diseases are limited, and are only beginning to be understood (Wu et al., 2021; Zhang et al., 2021). Since, there is evidence that secretory metabolites of commensal bacteria can directly modulate SC development (Kumar et al., 2020), it would be interesting to assess how agedependent gut metabolome changes affect the oxi-inflammatory stress in the gut, and whether these changes could be correlated with increased SC burden in the intestine (Figure 4). Secondly, there is an acute dearth of information whether the effects of putative anti-cellular senescence agents or senolytics and senomorphics could be related or even dependent on the modulation of the gut microbiome. This is significant since a focus is on identifying/developing diet-based senolytics, but their relationship with the gut microbiome is not clear. Moreover, probiotic bacteria, which have been ardently augmented as modulators of gut dysbiosis (Gagliardi et al., 2018), are yet to be conclusively assessed for their anticellular senescence attributes per se (Figure 4). Thirdly, it is essential to analyze the biological impact of senescent intestinal tissue cells in directly influencing the microflora composition as well as functional homeostasis during aging (Figure 4). This may be tested by studying the effects of SC and their secretome on the growth and abundance of specific microbial populations or a milieu of different representative microbes. Finally, we know the impact of microbiota in the development and maturation of immune system, and thus it is reasonable to speculate that strategies targeting gut dysbiosis could also improve immune functions during aging which may help counter SC burden and SASP development. However, as of now, this is purely speculative and further research is required to comprehend the relationship between age-related dysbiosis and immunosenescence vis-à-vis cellular senescence (Bosco and Noti, 2021).

\section{Conclusions}

It is accepted that gut microbiota and human aging are intimately linked. However, our understanding of the aging process per se is yet incomplete and is being progressively deciphered. Despite its limitations, cellular senescence-based understanding of aging is currently the focus of aging research (Bhatia-Dey et al., 2016), and which, combined with immunosenescence and gut dysbiosis form the major known contributors to aging (Figure 5). Given this emerging paradigm, it is essential that the gut microbiome be investigated in the emerging purview of cellular senescence, SASP and immunosenescence, which bears the promise to identify new therapeutic targets for microbiome oriented anti-aging interventions. 


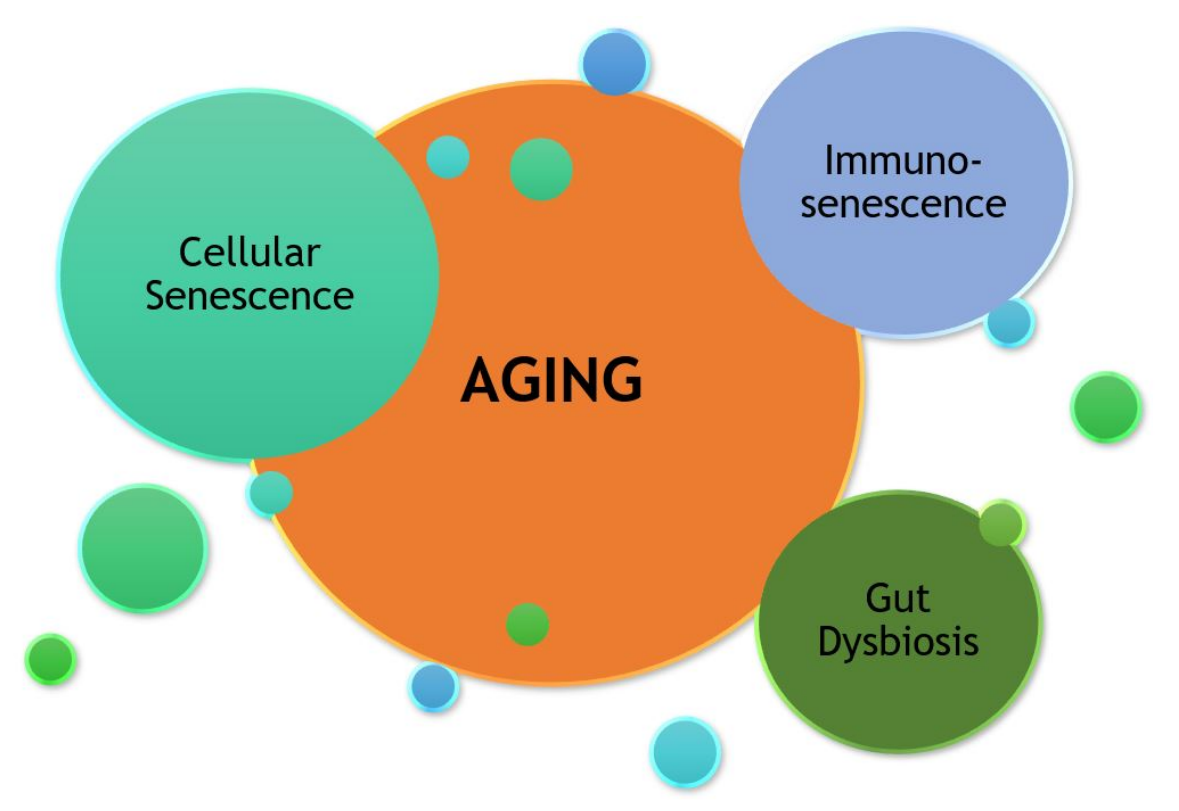

Figure 5. Cellular senescence, gut dysbiosis and immunosenescence are the major contributors to aging and age-related disorders.

\section{Funding sources}

This work was supported by grant from the Department of Science \& Technology, Government of India under the INSPIRE Faculty scheme (grant no. IFA17-LSPA79).

Declarations of interest: none

\section{References:}

Abrahamse, S.L., Pool-Zobel, B.L., and Rechkemmer, G. (1999). Potential of short chain fatty acids to modulate the induction of DNA damage and changes in the intracellular calcium concentration by oxidative stress in isolated rat distal colon cells. Carcinogenesis 20, 629-634. 10.1093/carcin/20.4.629.

Agus, A., Clément, K., and Sokol, H. (2021). Gut microbiota-derived metabolites as central regulators in metabolic disorders. Gut 70, 1174. 10.1136/gutjnl-2020-323071.

Almugadam, B.S., Liu, Y., Chen, S.-m., Wang, C.-h., Shao, C.-y., Ren, B.-w., and Tang, L. (2020). Alterations of Gut Microbiota in Type 2 Diabetes Individuals and the Confounding Effect of Antidiabetic Agents. Journal of Diabetes Research 2020, 7253978. 10.1155/2020/7253978.

Anand, R., Song, Y., Garg, S., Girotra, M., Sinha, A., Sivaraman, A., Phillips, L., and Dutta, S.K. (2017). Effect of Aging on the Composition of Fecal Microbiota in Donors for FMT and Its Impact on Clinical Outcomes. Dig Dis Sci 62, 1002-1008. 10.1007/s10620-0174449-6.

Andersen, K., Kesper, M.S., Marschner, J.A., Konrad, L., Ryu, M., Kumar Vr, S., Kulkarni, O.P., Mulay, S.R., Romoli, S., Demleitner, J., et al. (2017). Intestinal Dysbiosis, Barrier Dysfunction, and Bacterial Translocation Account for CKD-Related Systemic Inflammation. Journal of the American Society of Nephrology 28, 76. 10.1681/ASN.2015111285.

Baker, D.J., Childs, B.G., Durik, M., Wijers, M.E., Sieben, C.J., Zhong, J., Saltness, R.A., Jeganathan, K.B., Verzosa, G.C., Pezeshki, A., et al. (2016). Naturally occurring 
p16(Ink4a)-positive cells shorten healthy lifespan. Nature 530, 184-189.

10.1038/nature16932.

Baker, D.J., Wijshake, T., Tchkonia, T., LeBrasseur, N.K., Childs, B.G., van de Sluis, B., Kirkland, J.L., and van Deursen, J.M. (2011). Clearance of p16Ink4a-positive senescent cells delays ageing-associated disorders. Nature 479, 232-236. 10.1038/nature10600.

Bana, B., and Cabreiro, F. (2019). The Microbiome and Aging. Annual Review of Genetics 53, 239-261. 10.1146/annurev-genet-112618-043650.

Bhatia-Dey, N., Kanherkar, R.R., Stair, S.E., Makarev, E.O., and Csoka, A.B. (2016). Cellular Senescence as the Causal Nexus of Aging. Front Genet 7, 13. 10.3389/fgene.2016.00013.

Borghesan, M., Hoogaars, W.M.H., Varela-Eirin, M., Talma, N., and Demaria, M. (2020). A Senescence-Centric View of Aging: Implications for Longevity and Disease. Trends Cell Biol 30, 777-791. 10.1016/j.tcb.2020.07.002.

Bosco, N., and Noti, M. (2021). The aging gut microbiome and its impact on host immunity. Genes \& Immunity. 10.1038/s41435-021-00126-8.

Buford, T.W. (2017). (Dis)Trust your gut: the gut microbiome in age-related inflammation, health, and disease. Microbiome 5, 80. 10.1186/s40168-017-0296-0.

Bulua, A.C., Simon, A., Maddipati, R., Pelletier, M., Park, H., Kim, K.Y., Sack, M.N., Kastner, D.L., and Siegel, R.M. (2011). Mitochondrial reactive oxygen species promote production of proinflammatory cytokines and are elevated in TNFR1-associated periodic syndrome (TRAPS). J Exp Med 208, 519-533. 10.1084/jem.20102049.

Burton, D.G.A., and Stolzing, A. (2018). Cellular senescence: Immunosurveillance and future immunotherapy. Ageing Res Rev 43, 17-25. 10.1016/j.arr.2018.02.001.

Cai, Y., Zhou, H., Zhu, Y., Sun, Q., Ji, Y., Xue, A., Wang, Y., Chen, W., Yu, X., Wang, L., et al. (2020). Elimination of senescent cells by $\beta$-galactosidase-targeted prodrug attenuates inflammation and restores physical function in aged mice. Cell Research 30, 574-589. 10.1038/s41422-020-0314-9.

Campisi, J. (1997). The biology of replicative senescence. Eur J Cancer 33, 703-709. 10.1016/s0959-8049(96)00058-5.

Campisi, J. (2005). Senescent cells, tumor suppression, and organismal aging: good citizens, bad neighbors. Cell 120, 513-522. 10.1016/j.cell.2005.02.003.

Cardenas, K., Dao, V., Hurez, V., and Curiel, T. (2014). Age-related gut dysbiosis contributes to age-related colitis and colon cancer risk and appears B7-H1-dependent. (HUM8P.352). The Journal of Immunology 192, 185.127.

Chang, P.V., Hao, L., Offermanns, S., and Medzhitov, R. (2014). The microbial metabolite butyrate regulates intestinal macrophage function via histone deacetylase inhibition. Proceedings of the National Academy of Sciences 111, 2247. 10.1073/pnas.1322269111.

Chen, K., Shen, W., Zhang, Z., Xiong, F., Ouyang, Q., and Luo, C. (2020a). Age-dependent decline in stress response capacity revealed by proteins dynamics analysis. Scientific Reports 10, 15211. 10.1038/s41598-020-72167-4.

Chen, T.-J., Feng, Y., Liu, T., Wu, T.-T., Chen, Y.-J., Li, X., Li, Q., and Wu, Y.-C. (2020b). Fisetin Regulates Gut Microbiota and Exerts Neuroprotective Effect on Mouse Model of Parkinson's Disease. Frontiers in Neuroscience 14. 10.3389/fnins.2020.549037.

Chen, Z., Trotman, L.C., Shaffer, D., Lin, H.K., Dotan, Z.A., Niki, M., Koutcher, J.A., Scher, H.I., Ludwig, T., Gerald, W., et al. (2005). Crucial role of p53-dependent cellular senescence in suppression of Pten-deficient tumorigenesis. Nature 436, 725-730. 10.1038/nature03918.

Childs, B.G., Baker, D.J., Kirkland, J.L., Campisi, J., and van Deursen, J.M. (2014). Senescence and apoptosis: dueling or complementary cell fates? EMBO Rep 15, 11391153. 10.15252/embr.201439245. 
Chung, H.-J., Lee, H., Na, G., Jung, H., Kim, D.-G., Shin, S.-I., Jung, S.-E., Choi, I.-D., Lee, J.-H., Sim, J.-H., and Choi, H.-K. (2020). Metabolic and Lipidomic Profiling of Vegetable Juices Fermented with Various Probiotics. Biomolecules 10, 725. 10.3390/biom 10050725 .

Chung, H., Pamp, S.J., Hill, J.A., Surana, N.K., Edelman, S.M., Troy, E.B., Reading, N.C., Villablanca, E.J., Wang, S., Mora, J.R., et al. (2012). Gut immune maturation depends on colonization with a host-specific microbiota. Cell 149, 1578-1593. 10.1016/j.cell.2012.04.037.

Claesson, M.J., Cusack, S., Sullivan, O., Greene-Diniz, R., de Weerd, H., Flannery, E., Marchesi, J.R., Falush, D., Dinan, T., Fitzgerald, G., et al. (2011). Composition, variability, and temporal stability of the intestinal microbiota of the elderly. Proceedings of the National Academy of Sciences 108, 4586. 10.1073/pnas.1000097107.

Clarke, G., Stilling, R.M., Kennedy, P.J., Stanton, C., Cryan, J.F., and Dinan, T.G. (2014). Minireview: Gut microbiota: the neglected endocrine organ. Mol Endocrinol 28, 12211238. 10.1210/me.2014-1108.

Colavitti, R., and Finkel, T. (2005). Reactive oxygen species as mediators of cellular senescence. IUBMB Life 57, 277-281. 10.1080/15216540500091890.

Colldén, H., Landin, A., Wallenius, V., Elebring, E., Fändriks, L., Nilsson, M.E., Ryberg, H., Poutanen, M., Sjögren, K., Vandenput, L., and Ohlsson, C. (2019). The gut microbiota is a major regulator of androgen metabolism in intestinal contents. American Journal of Physiology-Endocrinology and Metabolism 317, E1182-E1192. 10.1152/ajpendo.00338.2019.

Cui, X., Ye, L., Li, J., Jin, L., Wang, W., Li, S., Bao, M., Wu, S., Li, L., Geng, B., et al. (2018). Metagenomic and metabolomic analyses unveil dysbiosis of gut microbiota in chronic heart failure patients. Sci Rep 8, 635. 10.1038/s41598-017-18756-2.

D'Alessandro, G., Antonangeli, F., Marrocco, F., Porzia, A., Lauro, C., Santoni, A., and Limatola, C. (2020). Gut microbiota alterations affect glioma growth and innate immune cells involved in tumor immunosurveillance in mice. Eur J Immunol 50, 705-711. 10.1002/eji.201948354.

D’Amato, A., Di Cesare Mannelli, L., Lucarini, E., Man, A.L., Le Gall, G., Branca, J.J.V., Ghelardini, C., Amedei, A., Bertelli, E., Regoli, M., et al. (2020). Faecal microbiota transplant from aged donor mice affects spatial learning and memory via modulating hippocampal synaptic plasticity- and neurotransmission-related proteins in young recipients. Microbiome 8, 140. 10.1186/s40168-020-00914-w.

Daisley, B.A., Chanyi, R.M., Abdur-Rashid, K., Al, K.F., Gibbons, S., Chmiel, J.A., Wilcox, H., Reid, G., Anderson, A., Dewar, M., et al. (2020). Abiraterone acetate preferentially enriches for the gut commensal Akkermansia muciniphila in castrate-resistant prostate cancer patients. Nature Communications 11, 4822. 10.1038/s41467-020-18649-5.

Das, B., Ghosh, T.S., Kedia, S., Rampal, R., Saxena, S., Bag, S., Mitra, R., Dayal, M., Mehta, O., Surendranath, A., et al. (2018). Analysis of the Gut Microbiome of Rural and Urban Healthy Indians Living in Sea Level and High Altitude Areas. Sci. Rep. 8, 10104. 10.1038/s41598-018-28550-3.

Das, T., Jayasudha, R., Chakravarthy, S., Prashanthi, G.S., Bhargava, A., Tyagi, M., Rani, P.K., Pappuru, R.R., Sharma, S., and Shivaji, S. (2021). Alterations in the gut bacterial microbiome in people with type 2 diabetes mellitus and diabetic retinopathy. Scientific Reports 11, 2738. 10.1038/s41598-021-82538-0.

de Magalhães, J.P., and Passos, J.F. (2018). Stress, cell senescence and organismal ageing. Mechanisms of Ageing and Development 170, 2-9. https://doi.org/10.1016/j.mad.2017.07.001. 
De Marco, S., Sichetti, M., Muradyan, D., Piccioni, M., Traina, G., Pagiotti, R., and Pietrella, D. (2018). Probiotic Cell-Free Supernatants Exhibited Anti-Inflammatory and Antioxidant Activity on Human Gut Epithelial Cells and Macrophages Stimulated with LPS. Evidence-Based Complementary and Alternative Medicine 2018, 1756308. $10.1155 / 2018 / 1756308$.

DeGruttola, A.K., Low, D., Mizoguchi, A., and Mizoguchi, E. (2016). Current Understanding of Dysbiosis in Disease in Human and Animal Models. Inflamm Bowel Dis 22, 1137 1150. 10.1097/MIB.0000000000000750.

Demaria, M., Ohtani, N., Youssef, S.A., Rodier, F., Toussaint, W., Mitchell, J.R., Laberge, R.M., Vijg, J., Van Steeg, H., Dollé, M.E., et al. (2014). An essential role for senescent cells in optimal wound healing through secretion of PDGF-AA. Dev Cell 31, 722-733. 10.1016/j.devcel.2014.11.012.

Dolgin, E. (2020). Send in the senolytics. Nature Biotechnology 38, 1371-1377. 10.1038/s41587-020-00750-1.

Dominguez-Bello, M.G., Costello, E.K., Contreras, M., Magris, M., Hidalgo, G., Fierer, N., and Knight, R. (2010). Delivery mode shapes the acquisition and structure of the initial microbiota across multiple body habitats in newborns. Proc. Natl. Acad. Sci. U. S. A. 107, 11971-11975. 10.1073/pnas.1002601107.

Donaldson, D.S., Pollock, J., Vohra, P., Stevens, M.P., and Mabbott, N.A. (2020). Microbial Stimulation Reverses the Age-Related Decline in M Cells in Aged Mice. iScience 23, 101147. 10.1016/j.isci.2020.101147.

Dos Santos, A.S., de Albuquerque, T.M.R., de Brito Alves, J.L., and de Souza, E.L. (2019). Effects of Quercetin and Resveratrol on in vitro Properties Related to the Functionality of Potentially Probiotic Lactobacillus Strains. Front Microbiol 10, 2229-2229. 10.3389/fmicb.2019.02229.

Ebert, M.N., Klinder, A., Peters, W.H., Schäferhenrich, A., Sendt, W., Scheele, J., and PoolZobel, B.L. (2003). Expression of glutathione S-transferases (GSTs) in human colon cells and inducibility of GSTM2 by butyrate. Carcinogenesis 24, 1637-1644. 10.1093/carcin/bgg122.

Elderman, M., Sovran, B., Hugenholtz, F., Graversen, K., Huijskes, M., Houtsma, E., Belzer, C., Boekschoten, M., de Vos, P., Dekker, J., et al. (2017). The effect of age on the intestinal mucus thickness, microbiota composition and immunity in relation to sex in mice. PloS one 12, e0184274-e0184274. 10.1371/journal.pone.0184274.

Eloe-Fadrosh, E.A., and Rasko, D.A. (2013). The human microbiome: from symbiosis to pathogenesis. Annu Rev Med 64, 145-163. 10.1146/annurev-med-010312-133513.

Epel, E.S., Blackburn, E.H., Lin, J., Dhabhar, F.S., Adler, N.E., Morrow, J.D., and Cawthon, R.M. (2004). Accelerated telomere shortening in response to life stress. Proc Natl Acad Sci U S A 101, 17312-17315. 10.1073/pnas.0407162101.

Ferreira, R.M., Pereira-Marques, J., Pinto-Ribeiro, I., Costa, J.L., Carneiro, F., Machado, J.C., and Figueiredo, C. (2018). Gastric microbial community profiling reveals a dysbiotic cancer-associated microbiota. Gut 67, 226. 10.1136/gutjnl-2017-314205.

Ferreiro, A., Crook, N., Gasparrini, A.J., and Dantas, G. (2018). Multiscale Evolutionary Dynamics of Host-Associated Microbiomes. Cell 172, 1216-1227. 10.1016/j.cell.2018.02.015.

Fransen, F., van Beek, A.A., Borghuis, T., Aidy, S.E., Hugenholtz, F., van der Gaast - de Jongh, C., Savelkoul, H.F.J., De Jonge, M.I., Boekschoten, M.V., Smidt, H., et al. (2017). Aged Gut Microbiota Contributes to Systemical Inflammaging after Transfer to Germ-Free Mice. Frontiers in Immunology 8. 10.3389/fimmu.2017.01385.

Frias-Lopez, J., Shi, Y., Tyson, G.W., Coleman, M.L., Schuster, S.C., Chisholm, S.W., and Delong, E.F. (2008). Microbial community gene expression in ocean surface waters. 
Proceedings of the National Academy of Sciences of the United States of America 105, 3805-3810. 10.1073/pnas.0708897105.

Gagliardi, A., Totino, V., Cacciotti, F., Iebba, V., Neroni, B., Bonfiglio, G., Trancassini, M., Passariello, C., Pantanella, F., and Schippa, S. (2018). Rebuilding the Gut Microbiota Ecosystem. Int J Environ Res Public Health 15, 1679. 10.3390/ijerph15081679.

Galkin, F., Mamoshina, P., Aliper, A., Putin, E., Moskalev, V., Gladyshev, V.N., and Zhavoronkov, A. (2020). Human Gut Microbiome Aging Clock Based on Taxonomic Profiling and Deep Learning. iScience 23, 101199. 10.1016/j.isci.2020.101199.

Gems, D., and Partridge, L. (2013). Genetics of longevity in model organisms: debates and paradigm shifts. Annu. Rev. Physiol. 75, 621-644. 10.1146/annurev-physiol-030212183712.

Gervason, S., Napoli, M., Dreux-Zhiga, A., Lazzarelli, C., Garcier, S., Briand, A., Albouy, M., Thepot, A., Berthon, J.Y., and Filaire, E. (2019). Attenuation of negative effects of senescence in human skin using an extract from Sphingomonas hydrophobicum: development of new skin care solution. Int J Cosmet Sci 41, 391-397. 10.1111/ics.12534.

Goedert, J.J., Jones, G., Hua, X., Xu, X., Yu, G., Flores, R., Falk, R.T., Gail, M.H., Shi, J., Ravel, J., and Feigelson, H.S. (2015). Investigation of the association between the fecal microbiota and breast cancer in postmenopausal women: a population-based case-control pilot study. J Natl Cancer Inst 107. 10.1093/jnci/djv147.

Goodrich, J.K., Davenport, E.R., Waters, J.L., Clark, A.G., and Ley, R.E. (2016). Crossspecies comparisons of host genetic associations with the microbiome. Science 352, 532535. 10.1126/science.aad9379.

Guigoz, Y., Rochat, F., Perruisseau-Carrier, G., Rochat, I., and Schiffrin, E.J. (2002). Effects of oligosaccharide on the faecal flora and non-specific immune system in elderly people. Nutrition Research 22, 13-25. https://doi.org/10.1016/S0271-5317(01)00354-2.

Gurung, M., Li, Z., You, H., Rodrigues, R., Jump, D.B., Morgun, A., and Shulzhenko, N. (2020). Role of gut microbiota in type 2 diabetes pathophysiology. EBioMedicine 51, 102590-102590. 10.1016/j.ebiom.2019.11.051.

Half, E., Keren, N., Reshef, L., Dorfman, T., Lachter, I., Kluger, Y., Reshef, N., Knobler, H., Maor, Y., Stein, A., et al. (2019). Fecal microbiome signatures of pancreatic cancer patients. Scientific Reports 9, 16801. 10.1038/s41598-019-53041-4.

He, S., and Sharpless, N.E. (2017). Senescence in Health and Disease. Cell 169, 1000-1011. 10.1016/j.cell.2017.05.015.

Herbig, U., Ferreira, M., Condel, L., Carey, D., and Sedivy, J.M. (2006). Cellular senescence in aging primates. Science 311, 1257. 10.1126/science.1122446.

Herranz, N., and Gil, J. (2018). Mechanisms and functions of cellular senescence. The Journal of clinical investigation 128, 1238-1246. 10.1172/JCI95148.

Hickson, L.J., Langhi Prata, L.G.P., Bobart, S.A., Evans, T.K., Giorgadze, N., Hashmi, S.K., Herrmann, S.M., Jensen, M.D., Jia, Q., Jordan, K.L., et al. (2019). Senolytics decrease senescent cells in humans: Preliminary report from a clinical trial of Dasatinib plus Quercetin in individuals with diabetic kidney disease. EBioMedicine 47, 446-456. https://doi.org/10.1016/j.ebiom.2019.08.069.

Hopkins, M.J., and Macfarlane, G.T. (2002). Changes in predominant bacterial populations in human faeces with age and with Clostridium difficile infection. J. Med. Microbiol. 51, 448-454. 10.1099/0022-1317-51-5-448.

Iatsenko, I., Boquete, J.P., and Lemaitre, B. (2018). Microbiota-Derived Lactate Activates Production of Reactive Oxygen Species by the Intestinal NADPH Oxidase Nox and Shortens Drosophila Lifespan. Immunity 49, 929-942.e925. 10.1016/j.immuni.2018.09.017. 
Idda, M.L., McClusky, W.G., Lodde, V., Munk, R., Abdelmohsen, K., Rossi, M., and Gorospe, M. (2020). Survey of senescent cell markers with age in human tissues. Aging 12, 4052-4066. 10.18632/aging.102903.

Jain, A., Li, X.H., and Chen, W.N. (2019). An untargeted fecal and urine metabolomics analysis of the interplay between the gut microbiome, diet and human metabolism in Indian and Chinese adults. Scientific Reports 9, 9191. 10.1038/s41598-019-45640-y.

Jeong, J.-J., Kim, K.-A., Jang, S.-E., Woo, J.-Y., Han, M.J., and Kim, D.-H. (2015a). Orally administrated Lactobacillus pentosus var. plantarum C29 ameliorates age-dependent colitis by inhibiting the nuclear factor-kappa B signaling pathway via the regulation of lipopolysaccharide production by gut microbiota. PloS one 10, e $0116533-\mathrm{e} 0116533$. 10.1371/journal.pone.0116533.

Jeong, J.J., Kim, K.A., Hwang, Y.J., Han, M.J., and Kim, D.H. (2016). Anti-inflammaging effects of Lactobacillus brevis OW38 in aged mice. Benef Microbes 7, 707-718. $10.3920 / \mathrm{bm} 2016.0016$.

Jeong, J.J., Woo, J.Y., Ahn, Y.T., Shim, J.H., Huh, C.S., Im, S.H., Han, M.J., and Kim, D.H. (2015b). The probiotic mixture IRT5 ameliorates age-dependent colitis in rats. Int Immunopharmacol 26, 416-422. 10.1016/j.intimp.2015.04.021.

Jie, Z., Xia, H., Zhong, S.L., Feng, Q., Li, S., Liang, S., Zhong, H., Liu, Z., Gao, Y., Zhao, H., et al. (2017). The gut microbiome in atherosclerotic cardiovascular disease. Nat Commun 8, 845. 10.1038/s41467-017-00900-1.

Jørgensen, S.M.D., Rubak, T.M.M., Damsgaard, E.M., Dahlerup, J.F., and Hvas, C.L. (2020). Faecal microbiota transplantation as a home therapy to frail older people. Age and Ageing 49, 1093-1096. 10.1093/ageing/afaa073.

Justice, J.N., Nambiar, A.M., Tchkonia, T., LeBrasseur, N.K., Pascual, R., Hashmi, S.K., Prata, L., Masternak, M.M., Kritchevsky, S.B., Musi, N., and Kirkland, J.L. (2019). Senolytics in idiopathic pulmonary fibrosis: Results from a first-in-human, open-label, pilot study. EBioMedicine 40, 554-563. https://doi.org/10.1016/j.ebiom.2018.12.052.

Kamo, T., Akazawa, H., Suda, W., Saga-Kamo, A., Shimizu, Y., Yagi, H., Liu, Q., Nomura, S., Naito, A.T., Takeda, N., et al. (2017). Dysbiosis and compositional alterations with aging in the gut microbiota of patients with heart failure. PLoS One 12, e0174099. 10.1371/journal.pone.0174099.

Kaoutari, A.E., Armougom, F., Gordon, J.I., Raoult, D., and Henrissat, B. (2013). The abundance and variety of carbohydrate-active enzymes in the human gut microbiota. Nature Reviews Microbiology 11, 497-504. 10.1038/nrmicro3050.

Karlsson, F.H., Fåk, F., Nookaew, I., Tremaroli, V., Fagerberg, B., Petranovic, D., Bäckhed, F., and Nielsen, J. (2012). Symptomatic atherosclerosis is associated with an altered gut metagenome. Nat Commun 3, 1245. 10.1038/ncomms2266.

Kim, M., and Benayoun, B.A. (2020). The microbiome: An emerging key player in aging and longevity. Translational Medicine of Aging 4, 103-116. https://doi.org/10.1016/j.tma.2020.07.004.

Kim, S.R., Jiang, K., Ferguson, C.M., Tang, H., Chen, X., Zhu, X., Hickson, L.J., Tchkonia, T., Kirkland, J.L., and Lerman, L.O. (2020). Transplanted senescent renal scattered tubular-like cells induce injury in the mouse kidney. American Journal of PhysiologyRenal Physiology 318, F1167-F1176. 10.1152/ajprenal.00535.2019.

Kirkland, J.L., and Tchkonia, T. (2020). Senolytic drugs: from discovery to translation. J Intern Med 288, 518-536. 10.1111/joim.13141.

Kong, F., Hua, Y., Zeng, B., Ning, R., Li, Y., and Zhao, J. (2016). Gut microbiota signatures of longevity. Curr. Biol. 26, R832-r833. 10.1016/j.cub.2016.08.015. 
Krishnamurthy, J., Torrice, C., Ramsey, M.R., Kovalev, G.I., Al-Regaiey, K., Su, L., and Sharpless, N.E. (2004). Ink4a/Arf expression is a biomarker of aging. J Clin Invest 114, 1299-1307. 10.1172/jci22475.

Kumar, R., Sharma, A., Gupta, M., Padwad, Y., and Sharma, R. (2020). Cell-Free Culture Supernatant of Probiotic Lactobacillus fermentum Protects Against H(2)O(2)-Induced Premature Senescence by Suppressing ROS-Akt-mTOR Axis in Murine Preadipocytes. Probiotics Antimicrob Proteins 12, 563-576. 10.1007/s12602-019-09576-Z.

Kumar, R., Sharma, A., Kumari, A., Gulati, A., Padwad, Y., and Sharma, R. (2019a). Epigallocatechin gallate suppresses premature senescence of preadipocytes by inhibition of PI3K/Akt/mTOR pathway and induces senescent cell death by regulation of Bax/Bcl2 pathway. Biogerontology 20, 171-189. 10.1007/s10522-018-9785-1.

Kumar, S., Suman, S., Fornace, A.J., and Datta, K. (2019b). Intestinal stem cells acquire premature senescence and senescence associated secretory phenotype concurrent with persistent DNA damage after heavy ion radiation in mice. Aging 11, 4145-4158. 10.18632/aging.102043.

Kundu, P., Lee, H.U., Garcia-Perez, I., Tay, E.X.Y., Kim, H., Faylon, L.E., Martin, K.A., Purbojati, R., Drautz-Moses, D.I., Ghosh, S., et al. (2019). Neurogenesis and prolongevity signaling in young germ-free mice transplanted with the gut microbiota of old mice. Science Translational Medicine 11, eaau4760. 10.1126/scitranslmed.aau4760.

Lee, H.-J., Lee, K.-E., Kim, J.-K., and Kim, D.-H. (2019). Suppression of gut dysbiosis by Bifidobacterium longum alleviates cognitive decline in 5XFAD transgenic and aged mice. Scientific Reports 9, 11814. 10.1038/s41598-019-48342-7.

Lee, J., Venna, V.R., Durgan, D.J., Shi, H., Hudobenko, J., Putluri, N., Petrosino, J., McCullough, L.D., and Bryan, R.M. (2020). Young versus aged microbiota transplants to germ-free mice: increased short-chain fatty acids and improved cognitive performance. Gut Microbes 12, 1-14. 10.1080/19490976.2020.1814107.

Li, Q., Chang, Y., Zhang, K., Chen, H., Tao, S., and Zhang, Z. (2020a). Implication of the gut microbiome composition of type 2 diabetic patients from northern China. Sci. Rep. 10, 5450. 10.1038/s41598-020-62224-3.

Li, Y., Ning, L., Yin, Y., Wang, R., Zhang, Z., Hao, L., Wang, B., Zhao, X., Yang, X., Yin, L., et al. (2020b). Age-related shifts in gut microbiota contribute to cognitive decline in aged rats. Aging (Albany NY) 12, 7801-7817. 10.18632/aging.103093.

Lin, J., Nie, X., Xiong, Y., Gong, Z., Chen, J., Chen, C., Huang, Y., and Liu, T. (2020). Fisetin regulates gut microbiota to decrease CCR9 $(+) / \mathrm{CXCR} 3(+) / \mathrm{CD} 4(+)$ T-lymphocyte count and IL-12 secretion to alleviate premature ovarian failure in mice. Am J Transl Res 12, 203-247.

Lin, R., Piao, M., and Song, Y. (2019). Dietary Quercetin Increases Colonic Microbial Diversity and Attenuates Colitis Severity in Citrobacter rodentium-Infected Mice. Front Microbiol 10, 1092-1092. 10.3389/fmicb.2019.01092.

Liu, Y., Sanoff, H.K., Cho, H., Burd, C.E., Torrice, C., Ibrahim, J.G., Thomas, N.E., and Sharpless, N.E. (2009). Expression of p16(INK4a) in peripheral blood T-cells is a biomarker of human aging. Aging Cell 8, 439-448. 10.1111/j.1474-9726.2009.00489.x.

López-Otín, C., Blasco, M.A., Partridge, L., Serrano, M., and Kroemer, G. (2013). The hallmarks of aging. Cell 153, 1194-1217. 10.1016/j.cell.2013.05.039.

Luedde, M., Winkler, T., Heinsen, F.A., Rühlemann, M.C., Spehlmann, M.E., Bajrovic, A., Lieb, W., Franke, A., Ott, S.J., and Frey, N. (2017). Heart failure is associated with depletion of core intestinal microbiota. ESC Heart Fail 4, 282-290. 10.1002/ehf2.12155.

Mariat, D., Firmesse, O., Levenez, F., Guimarăes, V.D., Sokol, H., Doré, J., Corthier, G., and Furet, J.P. (2009). The Firmicutes/Bacteroidetes ratio of the human microbiota changes with age. BMC Microbiol. 9, 123. 10.1186/1471-2180-9-123. 
Medawar, P.B. (1952). An unsolved problem of biology (Published for the college by H. K. Lewis).

Moorefield, E.C., Andres, S.F., Blue, R.E., Van Landeghem, L., Mah, A.T., Santoro, M.A., and Ding, S. (2017). Aging effects on intestinal homeostasis associated with expansion and dysfunction of intestinal epithelial stem cells. Aging 9, 1898-1915. 10.18632/aging.101279.

Muñoz-Espín, D., Cañamero, M., Maraver, A., Gómez-López, G., Contreras, J., MurilloCuesta, S., Rodríguez-Baeza, A., Varela-Nieto, I., Ruberte, J., Collado, M., and Serrano, M. (2013). Programmed cell senescence during mammalian embryonic development. Cell 155, 1104-1118. 10.1016/j.cell.2013.10.019.

Nakatsu, G., Li, X., Zhou, H., Sheng, J., Wong, S.H., Wu, W.K., Ng, S.C., Tsoi, H., Dong, Y., Zhang, N., et al. (2015). Gut mucosal microbiome across stages of colorectal carcinogenesis. Nat Commun 6, 8727. 10.1038/ncomms9727.

Nelson, G., Wordsworth, J., Wang, C., Jurk, D., Lawless, C., Martin-Ruiz, C., and von Zglinicki, T. (2012). A senescent cell bystander effect: senescence-induced senescence. Aging Cell 11, 345-349. 10.1111/j.1474-9726.2012.00795.x.

O'Toole, P.W., and Claesson, M.J. (2010). Gut microbiota: Changes throughout the lifespan from infancy to elderly. Int. Dairy J. 20, 281-291. https://doi.org/10.1016/j.idairyj.2009.11.010.

Oliphant, K., and Allen-Vercoe, E. (2019). Macronutrient metabolism by the human gut microbiome: major fermentation by-products and their impact on host health. Microbiome 7, 91. 10.1186/s40168-019-0704-8.

Ovadya, Y., Landsberger, T., Leins, H., Vadai, E., Gal, H., Biran, A., Yosef, R., Sagiv, A., Agrawal, A., Shapira, A., et al. (2018). Impaired immune surveillance accelerates accumulation of senescent cells and aging. Nature Communications 9, 5435. 10.1038/s41467-018-07825-3.

Parida, S., and Sharma, D. (2021). The Microbiome and Cancer: Creating Friendly Neighborhoods and Removing the Foes Within. Cancer Research 81, 790. 10.1158/00085472.CAN-20-2629.

Parkar, S.G., Trower, T.M., and Stevenson, D.E. (2013). Fecal microbial metabolism of polyphenols and its effects on human gut microbiota. Anaerobe 23, 12-19. https://doi.org/10.1016/j.anaerobe.2013.07.009.

Pereira, B.I., Devine, O.P., Vukmanovic-Stejic, M., Chambers, E.S., Subramanian, P., Patel, N., Virasami, A., Sebire, N.J., Kinsler, V., Valdovinos, A., et al. (2019). Senescent cells evade immune clearance via HLA-E-mediated NK and CD8+ T cell inhibition. Nature Communications 10, 2387. 10.1038/s41467-019-10335-5.

Poore, G.D., Kopylova, E., Zhu, Q., Carpenter, C., Fraraccio, S., Wandro, S., Kosciolek, T., Janssen, S., Metcalf, J., Song, S.J., et al. (2020). Microbiome analyses of blood and tissues suggest cancer diagnostic approach. Nature 579, 567-574. 10.1038/s41586-0202095-1.

Priya, S., and Blekhman, R. (2019). Population dynamics of the human gut microbiome: change is the only constant. Genome Biol. 20, 150. 10.1186/s13059-019-1775-3.

Renson, A., Mullan Harris, K., Dowd, J.B., Gaydosh, L., McQueen, M.B., Krauter, K.S., Shannahan, M., and Aiello, A.E. (2020). Early Signs of Gut Microbiome Aging: Biomarkers of Inflammation, Metabolism, and Macromolecular Damage in Young Adulthood. J Gerontol A Biol Sci Med Sci 75, 1258-1266. 10.1093/gerona/glaa122.

Report, H.o.L. (2021). Ageing: Science, Technology and Healthy Living. https://publications.parliament.uk/pa/ld5801/ldselect/ldsctech/183/183.pdf.

Riaz Rajoka, M.S., Zhao, H., Mehwish, H.M., Li, N., Lu, Y., Lian, Z., Shao, D., Jin, M., Li, Q., Zhao, L., and Shi, J. (2019). Anti-tumor potential of cell free culture supernatant of 
Lactobacillus rhamnosus strains isolated from human breast milk. Food Research International 123, 286-297. https://doi.org/10.1016/j.foodres.2019.05.002.

Rossi, T., Vergara, D., Fanini, F., Maffia, M., Bravaccini, S., and Pirini, F. (2020). Microbiota-Derived Metabolites in Tumor Progression and Metastasis. Int J Mol Sci 21, 5786. 10.3390/ijms21165786.

Saccon, T.D., Nagpal, R., Yadav, H., Cavalcante, M.B., Nunes, A.D.d.C., Schneider, A., Gesing, A., Hughes, B., Yousefzadeh, M., Tchkonia, T., et al. (2021). Senolytic Combination of Dasatinib and Quercetin Alleviates Intestinal Senescence and Inflammation and Modulates the Gut Microbiome in Aged Mice. The Journals of Gerontology: Series A. 10.1093/gerona/glab002.

Sager, R. (1991). Senescence as a mode of tumor suppression. Environ. Health Perspect. 93, 59-62. 10.1289/ehp.919359.

Salazar, N., Valdés-Varela, L., González, S., Gueimonde, M., and de Los Reyes-Gavilán, C.G. (2017). Nutrition and the gut microbiome in the elderly. Gut microbes 8, 82-97. 10.1080/19490976.2016.1256525.

Salekeen, R., Barua, J., Shaha, P.R., Islam, K.M.D., Islam, M.E., Billah, M.M., and Rahman, S.M.M. (2021). Marine phycocompound screening reveals a potential source of novel senotherapeutics. Journal of Biomolecular Structure and Dynamics, 1-15. 10.1080/07391102.2021.1877822.

Schluter, J., Peled, J.U., Taylor, B.P., Markey, K.A., Smith, M., Taur, Y., Niehus, R., Staffas, A., Dai, A., Fontana, E., et al. (2020). The gut microbiota is associated with immune cell dynamics in humans. Nature 588, 303-307. 10.1038/s41586-020-2971-8.

Sender, R., Fuchs, S., and Milo, R. (2016). Revised Estimates for the Number of Human and Bacteria Cells in the Body. PLoS Biol. 14, e1002533-e1002533. 10.1371/journal.pbio.1002533.

Sharma, R., Kapila, R., Dass, G., and Kapila, S. (2014). Improvement in Th1/Th2 immune homeostasis, antioxidative status and resistance to pathogenic E. coli on consumption of probiotic Lactobacillus rhamnosus fermented milk in aging mice. AGE 36, 9686. 10.1007/s11357-014-9686-4.

Sharma, R., Kumar, R., Sharma, A., Goel, A., and Padwad, Y. (2021). Long term consumption of green tea EGCG enhances healthspan and lifespan in mice by mitigating multiple aspects of cellular senescence in mitotic and post-mitotic tissues, gut dysbiosis and immunosenescence. bioRxiv, 2021.2001.2001.425058. 10.1101/2021.01.01.425058.

Sharma, R., Kumari, M., Kumari, A., Sharma, A., Gulati, A., Gupta, M., and Padwad, Y. (2019). Diet supplemented with phytochemical epigallocatechin gallate and probiotic Lactobacillus fermentum confers second generation synbiotic effects by modulating cellular immune responses and antioxidant capacity in aging mice. European Journal of Nutrition 58, 2943-2957. 10.1007/s00394-018-01890-6.

Sharma, R., and Padwad, Y. (2020a). Nutraceuticals-Based Immunotherapeutic Concepts and Opportunities for the Mitigation of Cellular Senescence and Aging: A Narrative Review. Ageing Res Rev 63, 101141. 10.1016/j.arr.2020.101141.

Sharma, R., and Padwad, Y. (2020b). Perspectives of the potential implications of polyphenols in influencing the interrelationship between oxi-inflammatory stress, cellular senescence and immunosenescence during aging. Trends in Food Science \& Technology 98, 41-52. https://doi.org/10.1016/j.tifs.2020.02.004.

Sharma, R., and Padwad, Y. (2020c). Plant-polyphenols based second-generation synbiotics: Emerging concepts, challenges, and opportunities. Nutrition 77, 110785. https://doi.org/10.1016/j.nut.2020.110785. 
Sharma, R., and Padwad, Y. (2020d). Probiotic bacteria as modulators of cellular senescence: emerging concepts and opportunities. Gut Microbes 11, 335-349.

10.1080/19490976.2019.1697148.

Shen, X., Miao, J., Wan, Q., Wang, S., Li, M., Pu, F., Wang, G., Qian, W., Yu, Q., Marotta, F., and He, F. (2018). Possible correlation between gut microbiota and immunity among healthy middle-aged and elderly people in southwest China. Gut Pathogens 10, 4. 10.1186/s13099-018-0231-3.

Shi, T., Bian, X., Yao, Z., Wang, Y., Gao, W., and Guo, C. (2020). Quercetin improves gut dysbiosis in antibiotic-treated mice. Food Funct 11, 8003-8013. 10.1039/d0fo01439g.

Smith, P., Willemsen, D., Popkes, M., Metge, F., Gandiwa, E., Reichard, M., and Valenzano, D.R. (2017). Regulation of life span by the gut microbiota in the short-lived African turquoise killifish. Elife 6. 10.7554/eLife.27014.

Smith, P.A. (2015). The tantalizing links between gut microbes and the brain. Nature 526, 312-314. 10.1038/526312a.

Sobhani, I., Bergsten, E., Couffin, S., Amiot, A., Nebbad, B., Barau, C., de'Angelis, N., Rabot, S., Canoui-Poitrine, F., Mestivier, D., et al. (2019). Colorectal cancer-associated microbiota contributes to oncogenic epigenetic signatures. Proceedings of the National Academy of Sciences 116, 24285-24295. 10.1073/pnas.1912129116.

Song, P., An, J., and Zou, M.-H. (2020). Immune Clearance of Senescent Cells to Combat Ageing and Chronic Diseases. Cells 9, 671. 10.3390/cells9030671.

Spallarossa, P., Altieri, P., Aloi, C., Garibaldi, S., Barisione, C., Ghigliotti, G., Fugazza, G., Barsotti, A., and Brunelli, C. (2009). Doxorubicin induces senescence or apoptosis in rat neonatal cardiomyocytes by regulating the expression levels of the telomere binding factors 1 and 2. Am J Physiol Heart Circ Physiol 297, H2169-2181. 10.1152/ajpheart.00068.2009.

Tang, W.H.W., Wang, Z., Fan, Y., Levison, B., Hazen, J.E., Donahue, L.M., Wu, Y., and Hazen, S.L. (2014). Prognostic value of elevated levels of intestinal microbe-generated metabolite trimethylamine-N-oxide in patients with heart failure: refining the gut hypothesis. J Am Coll Cardiol 64, 1908-1914. 10.1016/j.jacc.2014.02.617.

Tang, Z.-Z., Chen, G., Hong, Q., Huang, S., Smith, H.M., Shah, R.D., Scholz, M., and Ferguson, J.F. (2019). Multi-Omic Analysis of the Microbiome and Metabolome in Healthy Subjects Reveals Microbiome-Dependent Relationships Between Diet and Metabolites. Frontiers in Genetics 10. 10.3389/fgene.2019.00454.

Tchirkov, A., and Lansdorp, P.M. (2003). Role of oxidative stress in telomere shortening in cultured fibroblasts from normal individuals and patients with ataxia-telangiectasia. Human Molecular Genetics 12, 227-232. 10.1093/hmg/ddg023.

Thangaraju, M., Cresci, G.A., Liu, K., Ananth, S., Gnanaprakasam, J.P., Browning, D.D., Mellinger, J.D., Smith, S.B., Digby, G.J., Lambert, N.A., et al. (2009). GPR109A is a Gprotein-coupled receptor for the bacterial fermentation product butyrate and functions as a tumor suppressor in colon. Cancer Res 69, 2826-2832. 10.1158/0008-5472.Can-084466.

Thevaranjan, N., Puchta, A., Schulz, C., Naidoo, A., Szamosi, J.C., Verschoor, C.P., Loukov, D., Schenck, L.P., Jury, J., Foley, K.P., et al. (2017). Age-Associated Microbial Dysbiosis Promotes Intestinal Permeability, Systemic Inflammation, and Macrophage Dysfunction. Cell Host Microbe 21, 455-466.e454. 10.1016/j.chom.2017.03.002.

Tran, L., and Greenwood-Van Meerveld, B. (2013). Age-Associated Remodeling of the Intestinal Epithelial Barrier. The Journals of Gerontology: Series A 68, 1045-1056. 10.1093/gerona/glt106.

Tuttle, C.S.L., Waaijer, M.E.C., Slee-Valentijn, M.S., Stijnen, T., Westendorp, R., and Maier, A.B. (2020). Cellular senescence and chronological age in various human tissues: A 
systematic review and meta-analysis. Aging Cell 19, e13083.

https://doi.org/10.1111/acel.13083.

Uchida, R., Saito, Y., Nogami, K., Kajiyama, Y., Suzuki, Y., Kawase, Y., Nakaoka, T., Muramatsu, T., Kimura, M., and Saito, H. (2018). Epigenetic silencing of Lgr5 induces senescence of intestinal epithelial organoids during the process of aging. npj Aging and Mechanisms of Disease 4, 12. 10.1038/s41514-018-0031-5.

Vaiserman, A., Romanenko, M., Piven, L., Moseiko, V., Lushchak, O., Kryzhanovska, N., Guryanov, V., and Koliada, A. (2020). Differences in the gut Firmicutes to Bacteroidetes ratio across age groups in healthy Ukrainian population. BMC microbiology 20, 221221. 10.1186/s 12866-020-01903-7.

van Deursen, J.M. (2014). The role of senescent cells in ageing. Nature 509, 439-446. 10.1038 /nature13193.

Varela-Eirín, M., Carpintero-Fernández, P., Sánchez-Temprano, A., Varela-Vázquez, A., Paíno, C.L., Casado-Díaz, A., Continente, A.C., Mato, V., Fonseca, E., Kandouz, M., et al. (2020). Senolytic activity of small molecular polyphenols from olive restores chondrocyte redifferentiation and promotes a pro-regenerative environment in osteoarthritis. Aging 12, 15882-15905. 10.18632/aging.103801.

Vascellari, S., Palmas, V., Melis, M., Pisanu, S., Cusano, R., Uva, P., Perra, D., Madau, V., Sarchioto, M., Oppo, V., et al. (2020). Gut Microbiota and Metabolome Alterations Associated with Parkinson's Disease. mSystems 5, e00561-00520.

10.1128/mSystems.00561-20.

Vicente-Dueñas, C., Janssen, S., Oldenburg, M., Auer, F., González-Herrero, I., CasadoGarcía, A., Isidro-Hernández, M., Raboso-Gallego, J., Westhoff, P., Pandyra, A.A., et al. (2020). An intact gut microbiome protects genetically predisposed mice against leukemia. Blood 136, 2003-2017. 10.1182/blood.2019004381.

Wang, L.C., Pan, T.M., and Tsai, T.Y. (2018). Lactic acid bacteria-fermented product of green tea and Houttuynia cordata leaves exerts anti-adipogenic and anti-obesity effects. J Food Drug Anal 26, 973-984. 10.1016/j.jfda.2017.11.009.

Wang, Z., Klipfell, E., Bennett, B.J., Koeth, R., Levison, B.S., Dugar, B., Feldstein, A.E., Britt, E.B., Fu, X., Chung, Y.M., et al. (2011). Gut flora metabolism of phosphatidylcholine promotes cardiovascular disease. Nature 472, 57-63. 10.1038/nature09922.

Wei, W., Sun, W., Yu, S., Yang, Y., and Ai, L. (2016). Butyrate production from high-fiber diet protects against lymphoma tumor. Leuk Lymphoma 57, 2401-2408. 10.3109/10428194.2016.1144879.

Wilmanski, T., Diener, C., Rappaport, N., Patwardhan, S., Wiedrick, J., Lapidus, J., Earls, J.C., Zimmer, A., Glusman, G., Robinson, M., et al. (2021). Gut microbiome pattern reflects healthy ageing and predicts survival in humans. Nature Metabolism 3, 274-286. 10.1038/s42255-021-00348-0.

Wu, C.-S., Muthyala, S.D.V., Klemashevich, C., Ufondu, A.U., Menon, R., Chen, Z., Devaraj, S., Jayaraman, A., and Sun, Y. (2021). Age-dependent remodeling of gut microbiome and host serum metabolome in mice. Aging 13, 6330-6345. 10.18632/aging.202525.

Xu, M., Bradley, E.W., Weivoda, M.M., Hwang, S.M., Pirtskhalava, T., Decklever, T., Curran, G.L., Ogrodnik, M., Jurk, D., Johnson, K.O., et al. (2017). Transplanted Senescent Cells Induce an Osteoarthritis-Like Condition in Mice. J Gerontol A Biol Sci Med Sci 72, 780-785. 10.1093/gerona/glw154.

Xu, M., Pirtskhalava, T., Farr, J.N., Weigand, B.M., Palmer, A.K., Weivoda, M.M., Inman, C.L., Ogrodnik, M.B., Hachfeld, C.M., Fraser, D.G., et al. (2018). Senolytics improve 
physical function and increase lifespan in old age. Nature Medicine 24, 1246-1256. 10.1038/s41591-018-0092-9.

Yeoh, Y.K., Zuo, T., Lui, G.C.-Y., Zhang, F., Liu, Q., Li, A.Y., Chung, A.C., Cheung, C.P., Tso, E.Y., Fung, K.S., et al. (2021). Gut microbiota composition reflects disease severity and dysfunctional immune responses in patients with COVID-19. Gut 70, 698-706. 10.1136/gutjnl-2020-323020.

Yoshimoto, S., Loo, T.M., Atarashi, K., Kanda, H., Sato, S., Oyadomari, S., Iwakura, Y., Oshima, K., Morita, H., Hattori, M., et al. (2013). Obesity-induced gut microbial metabolite promotes liver cancer through senescence secretome. Nature 499, 97-101. 10.1038 /nature12347.

Yousefzadeh, M.J., Flores, R.R., Zhu, Y., Schmiechen, Z.C., Brooks, R.W., Trussoni, C.E., Cui, Y., Angelini, L., Lee, K.-A., McGowan, S.J., et al. (2021). An aged immune system drives senescence and ageing of solid organs. Nature. 10.1038/s41586-021-03547-7.

Yousefzadeh, M.J., Zhao, J., Bukata, C., Wade, E.A., McGowan, S.J., Angelini, L.A., Bank, M.P., Gurkar, A.U., McGuckian, C.A., Calubag, M.F., et al. (2020). Tissue specificity of senescent cell accumulation during physiologic and accelerated aging of mice. Aging Cell 19, e13094. https://doi.org/10.1111/acel.13094.

Zhang, X., Yang, Y., Su, J., Zheng, X., Wang, C., Chen, S., Liu, J., Lv, Y., Fan, S., Zhao, A., et al. (2020). Age-related compositional changes and correlations of gut microbiome, serum metabolome, and immune factor in rats. GeroScience. 10.1007/s11357-02000188-y.

Zhang, X., Yang, Y., Su, J., Zheng, X., Wang, C., Chen, S., Liu, J., Lv, Y., Fan, S., Zhao, A., et al. (2021). Age-related compositional changes and correlations of gut microbiome, serum metabolome, and immune factor in rats. GeroScience 43, 709-725. 10.1007/s11357-020-00188-y.

Zhang, X., Zhu, X., Sun, Y., Hu, B., Sun, Y., Jabbar, S., and Zeng, X. (2013). Fermentation in vitro of EGCG, GCG and EGCG3"Me isolated from Oolong tea by human intestinal microbiota. Food Research International 54, 1589-1595.

https://doi.org/10.1016/j.foodres.2013.10.005.

Zhang, Y., Unnikrishnan, A., Deepa, S.S., Liu, Y., Li, Y., Ikeno, Y., Sosnowska, D., Van Remmen, H., and Richardson, A. (2017). A new role for oxidative stress in aging: The accelerated aging phenotype in Sod1-/- mice is correlated to increased cellular senescence. Redox Biology 11, 30-37. https://doi.org/10.1016/j.redox.2016.10.014.

Zhu, Q., Gao, R., Zhang, Y., Pan, D., Zhu, Y., Zhang, X., Yang, R., Jiang, R., Xu, Y., and Qin, H. (2018). Dysbiosis signatures of gut microbiota in coronary artery disease. Physiol Genomics 50, 893-903. 10.1152/physiolgenomics.00070.2018.

Zhu, Y., Tchkonia, T., Pirtskhalava, T., Gower, A.C., Ding, H., Giorgadze, N., Palmer, A.K., Ikeno, Y., Hubbard, G.B., Lenburg, M., et al. (2015). The Achilles' heel of senescent cells: from transcriptome to senolytic drugs. Aging Cell 14, 644-658. 10.1111/acel.12344.

Zhuang, H., Cheng, L., Wang, Y., Zhang, Y.-K., Zhao, M.-F., Liang, G.-D., Zhang, M.-C., Li, Y.-G., Zhao, J.-B., Gao, Y.-N., et al. (2019). Dysbiosis of the Gut Microbiome in Lung Cancer. Front Cell Infect Microbiol 9, 112-112. 10.3389/fcimb.2019.00112.

Zierer, J., Jackson, M.A., Kastenmüller, G., Mangino, M., Long, T., Telenti, A., Mohney, R.P., Small, K.S., Bell, J.T., Steves, C.J., et al. (2018). The fecal metabolome as a functional readout of the gut microbiome. Nature Genetics 50, 790-795. 10.1038/s41588018-0135-7. 